\section{Effects of checkerboard pattern stimulation on evoked cortical responses in relation to check size and visual field*}

\author{
ROBERT G. EASON, University of North Carolina, Greensboro, N.C. 27412 \\ CARROLL T. WHITE, Navy Electronics Laboratory Center, San Diego, Calif. 92152 \\ and \\ NEIL BARTLETT, University of Arizona, Tucson, Ariz. 85721
}

Cortical evoked responses to checkerboard stimulation were studied as a function of check size and visual field. Stimuli were presented to the upper, central, and lower parts of the retina. Regardless of check size, largest potentials were obtained to lower-field stimulation; central-field stimulation yielded intermediate responses; upper-field stimulation produced the smallest responses. Maximal responses were obtained to intermediate check sizes during lower-field stimulation and to the smallest checks during upper-field stimulation. The results suggest that the cortical visual system is more responsive to patterned stimuli appearing in the lower viewing field than in the upper, but that the system may be relatively more sensitive to smaller objects appearing in the upper field.

Various studies have shown that the amplitude of the averaged evoked cortical response is related to the visual field in which the stimulus appears (Eason, Oden, \& White, 1967; Eason, White, \& Oden, 1967; Eason \& White, 1967) and to the pattern or contour of the stimulus (e.g., Harter \& White, 1968, 1970; Harter \& Suitt, 1970; Spehlmann, 1965; John et al, 1967; Rietveld et al, 1967). Using a small diffuse circular flash of light as a stimulus, Eason, White, \& Oden (1967) found that larger potentials are evoked by flashes appearing in the lower visual field than in the upper. This observation holds for retinal sites ranging in eccentricity from 10 to $60 \mathrm{deg}$ above and below the fovea. Related observations with patterned stimuli (black and white checkerboard squares) have been made by Jeffreys (1968), a complete summary of which appears in a report by. MacKay (1969). His data indicate that both the amplitude and waveform of the cortical responses are differentially affected by stimulation from within the upper and lower visual fields, the difference being dependent on the placement of the scalp electrode over the occipital area.

Studies by Harter \& White $(1968,1970)$ and others have shown that the size of black and white squares (i.e., contour density) comprising checkerboard pattern

*This study was supported in part by NSF Grant GB-7324 and by the Navy Electronics Laboratory Center. The statements contained herein are not to be construed as representing the views of the Navy Electronics Laboratory Center. The authors wish to express their appreciation to Mr. John Hoke for his technical assistance and to Miss Sandy Buck and Mr. Roger Hintze for serving as $S$ s and for their assistance in analyzing the data. on the evoked response as a function of

$$
S U B J E C T: \text { N.R.B. }
$$

retinal eccentricity and found the expected interaction between check size and eccentricity.

The present study manipulated check size in relation to stimulation of the visual system from within the upper, central, and lower fields in order to ascertain the interaction effects of check size and visual field on the evoked response. The purpose of these manipulations was to shed further light on the significance of the cortical evoked potential as it relates to the visual process and to serve as a further check on, as well as an extension of, the findings reported above.

\section{METHOD}

Five Ss participated in four experimental sessions each. A given session consisted of five runs, wherein a total of five check sizes and three visual field conditions were presented. Check size was varied across runs, visual field within runs. Order of presentation of check size was counterbalanced across sessions, visual field within each run. No S participated in more than one session on a given day.

The check stimuli consisted of black and white squares photographically reproduced onto $11 \times 14$ in. transparent sheets of film. Each stimulus display subtended a visual angle of $9 \mathrm{deg}, 35 \mathrm{~min}$ along both the horizontal and vertical dimensions. The checks composing the five displays$$
\text { CHECK S I Z E (M IN. OF ARC) }
$$

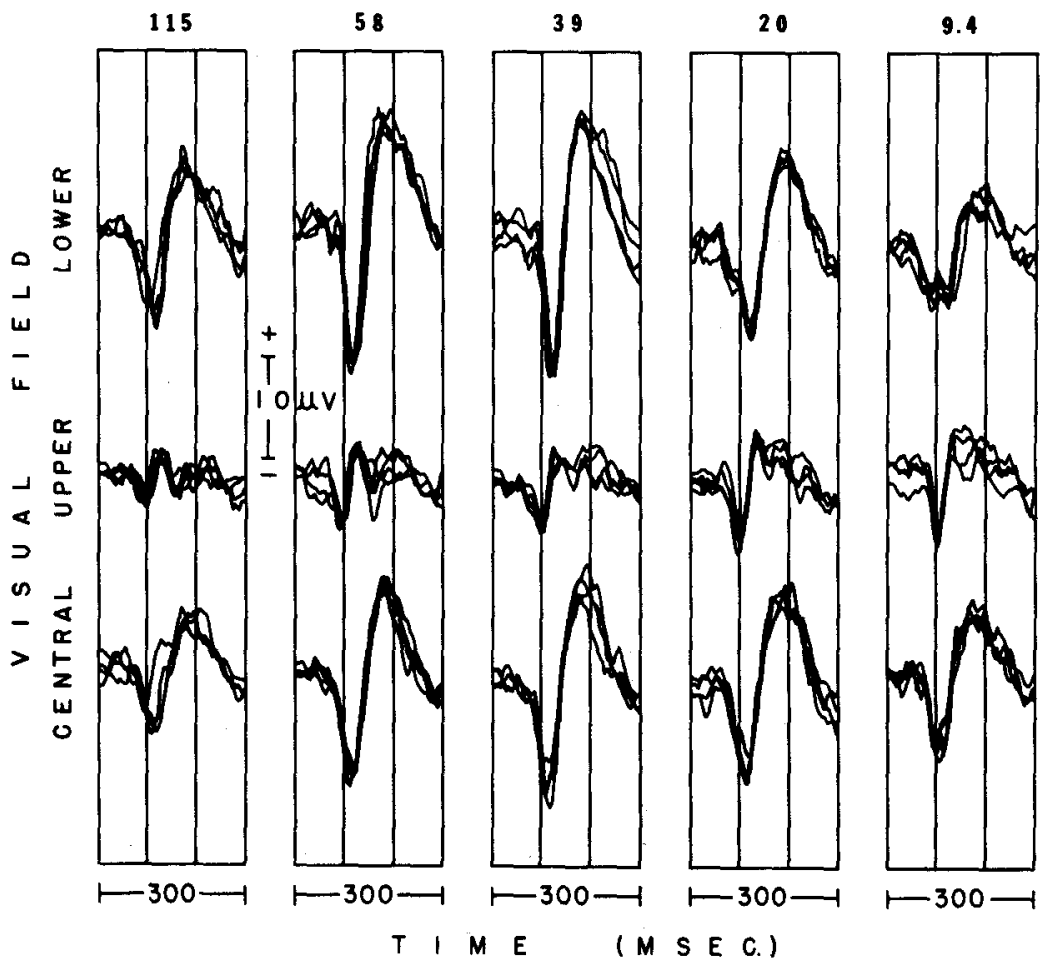

Fig. 1. Averaged evoked cortical responses to checkerboard stimulation for $S$ NRB. The responses are arranged by check size (columns) and visual field (rows). 


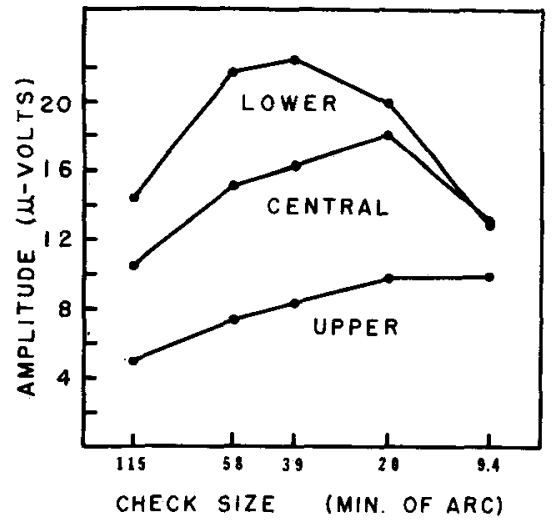

Fig. 2. Average amplitude of the major component of the evoked response $\left(P_{1}\right)$, based on the data of all Ss.

subtended visual angles of 9.4, 20,39, 58, and $115 \mathrm{~min}$ of arc along each side. During a run the displays were illuminated from the rear for 10 microseconds every $1.1 \mathrm{sec}$, using a Grass PS-2 photostimulator set at an intensity level of 4 . The $S$ viewed the displays binocularly at a distance of $137.2 \mathrm{~cm}$ from within a dark electrically shielded room.

During a given run, wherein one of the check stimuli was presented throughout, $S$ fixated the display at (1) the midpoint of the upper edge (lower-field stimulation), (2) the center (central-field stimulation), and (3) the midpoint of the lower edge (upper-field stimulation). The background illumination of the S's room and S's high luminance adaptation level (generated by the photostimulator flashes) made the room appear pitch black between flashes. To help $S$ maintain the appropriate fixation, two dimly illuminated grain-of-wheat bulbs were positioned, prior to each recording period, on either side of the display along a horizontal plane containing the point of fixation.

Evoked potentials were recorded monopolarly with an active scalp electrode attached along the midline $1 \mathrm{in}$. above the inion and with a "neutral" electrode attached to the right earlobe. The potentials were amplified with a Beckman-Offner Type R Dynagraph equipped with a $9806 \mathrm{~A}$ input complex and averaged with a TMC Computer of Average Transients (CAT), Model $400 \mathrm{C}$. Permanent records were obtained with a Moseley X-Y plotter. The averaged responses obtained in a given run, one for each of the three fixation conditions, were based on 50 flashes presented in counterbalanced order in blocks of 25.

\section{RESULTS}

The evoked potential records shown in Fig. 1 are representative of those obtained from all five Ss. The first major deflection is negative (N1), its onset latency being
$50-90 \mathrm{msec}$, depending on the experimental condition. The second and largest deflection is positive (P1) with an onset latency of 90-120 msec. It also varies with the experimental condition, particularly visual field. A third deflection (N2), with an onset latency of $120-180 \mathrm{msec}$, is clearly distinguishable, but thereafter the evoked response repidly attenuates, making it difficult to ascertain clearly the beginning and end of a second positive potential in most cases. The superimposed tracings of Fig. 1 show the high degree of consistency with which the evoked responses could be replicated for each experimental condition.

\section{Amplitude Effects}

To quantify the effects of check size and visual field on the amplitude of the evoked responses, the height of the largest deflection (P1) was measured, and the group data subjected to an analysis of variance. The average amplitude of P1 obtained for each experimental condition is plotted in Fig. 2. As the graphic functions suggest, the effects of check size, visual field, and their interactions were all highly significant ( $p<.01$ in every case).

With respect to visual field, much larger responses were obtained with lower-field stimulation than with upper, intermediate responses being obtained with central stimulation.

Concerning check size, the effects were dependent on where in the visual field the stimulus display was presented. Inverted $U$-shaped functions were obtained for lower- and central-field stimulation, largest responses being evoked when 39-min checks appeared in the lower field and 58-min checks in the central; an inverse monotonic relation was found for upper-field stimulation, largest responses being evoked by the smallest check (approximately $10 \mathrm{~min}$ of arc). The effects of check size were most pronounced for lower field stimulation, as is readily apparent in both Figs. 1 and 2.

\section{Latency and Waveform}

Responses evoked by upper-field stimulation differed from those evoked by both lower- and central-field stimulation, not only with respect to amplitude, but also with respect to latency and waveform. These differences are manifested in Fig. 1.

For every check size, Components N1 and $\mathrm{P} 1$ occurred sooner in those responses evoked by upper-field stimulation. The upper-lower field differences are dramatized in Fig. 1 by the fact that the responses are out of phase at approximately $115 \mathrm{msec}$ following the stimulus. This point in time corresponds to the peak latency of $\mathrm{N} 1$ of the lower field response and to $\mathrm{P} 1$ of the upper field response. The latencies obtained for central-field responses tended to fall between those obtained for upper- and lower-field responses.

With respect to waveform, the responses evoked by large checks presented in the upper field were clearly different in shape from those evoked by lower- and central-field stimulation, as well as from those evoked by small checks appearing in the upper field. As Fig. 1 shows, the upper-field large check size responses contained a second sinusoidal oscillation which was not present in responses obtained under any of the other conditions.

\section{DISCUSSION}

The inverted U-shaped relation between check size and evoked potential amplitude obtained for both central- and lower-field stimulation (Fig. 2) is consistent with, and further corroborates, the earlier findings of Harter (in press) and Harter \& White (1970). The inverse monotonic relation obtained in the present study for the upper-field condition (Fig. 2) may be the left side of an inverted U-shaped function which would have been revealed in its entirety had smaller checks been used in the experiment. Since the monotonic function in Fig. 2 shows signs of leveling off, we may surmise that the apex of the hypothesized inverted $U$ function would be generated by checks closely approximating the smallest ( $9.4 \mathrm{~min}$ ) used in this study.

In considering the finding that greater responses occur to lower- than to upper-field stimulation, regardless of check size, it should be noted in Fig. 2 that only for the stimulus display containing the smallest checks does the response amplitude to upper-field stimulation approach that of the lower field. The convergence of the two functions results primarily from a marked decrease in amplitude of the lower-field response to the smallest check size. Thus, it appears that except for relatively fine grain stimuli, a pattern appearing in the lower field acts as a more potent stimulus for eliciting evoked responses at the cortical level of the visual system than does the same pattern appearing in the upper field. Since man is a ground based creature, it is tempting to speculate that we are dealing with a mechanism which has evolved as a consequence of man's need to be particularly attuned and reactive to stimuli appearing in the lower field. This speculation may be tested indirectly by utilizing the pattem stimuli of the present experiment to ascertain whether differences similar to those observed for the evoked response also exist between the upper and lower fields with respect to visual acuity, threshold, reaction time, and other behavioral measures. 
The above discussion must be viewed with caution, as a second interpretation of the findings may be made on the basis of the topography of the visual cortex. Due to the spatial representation of the retina in the visual cortex, we may assume that the upper- and lower-field responses obtained in this study originated at distinctly different areas of the striate cortex within the calcarine fissure. Thus the potentials recorded between the scalp and earlobe may have been affected by differences in the volume conduction characteristics of the brain in relation to the loci of origin of the evoked responses. Perhaps greater attenuation occurred in the volume conductor in relation to the site of origin of the upper-field responses and that these responses at their site of origin were actually equal to or greater than the lower-field responses occurring in the calcarine fissure. Jeffreys (1968) has reported some electrode location data which suggests that the topographical factor may be important. Work is under way in our laboratory to test further this topographical hypothesis.

Even if the topographical explanation should have some validity in accounting for upper-lower field differences, it seems an unlikely candidate for explaining the effects of check size on evoked potential amplitude. There is no reason to assume that the effects are due to differences in the origin of the evoked response within the calcarine fissure, since exactly the same retinal area within each field is stimulated with each checkerboard stimulus display. It seems equally unlikely that the shift in maximal response amplitude from larger to smaller checks as the stimulus display is moved from the lower to the upper field (Fig. 2) is due to differences in the loci of origin of the responses.

The effects of check size may be more plausibly explained in terms of differences in the size of the centers of retinal receptive fields located in the retinal areas projecting respectively to the upper and lower visual fields. Harter \& White (1970) have built a rather strong case for the possibility that cortical evoked responses may be related to the size of receptive field centers, as reflected in the single unit responses of animals. Recently Harter (in press) obtained an interaction between check size and retinal eccentricity which was predicted from the hypothesis that evoked potentials and size of receptive field centers are related.

Assuming that the receptive field hypothesis has validity, the results depicted in Fig. 2 suggest that the receptive field centers located in that part of the retina stimulated from within the lower field have an average size of approximately $40 \mathrm{~min}$, whereas those which project to the upper field subtend about 10 min of visual angle. Why the retina projecting to the upper field should have smaller receptive-field centers than the area projecting to the lower field is not entirely clear. One may speculate that flying or rapidly moving objects appearing in the upper field must be detected while relatively far away if the organism is to respond appropriately to them. Thus, the part of the visual system projecting to the upper field may be particularly attuned to "specks in the sky" subtending less than $30 \mathrm{~min}$ of visual angle. On the other hand, ground objects which are sufficiently close to the organism to be potentially threatening generally may subtend somewhat larger visual angles and therefore that part of the visual system projecting to the lower field developed a relatively high degree of reactivity to objects subtending 30 or more minutes of visual angle.

\section{REFERENCES}

EASON, R. G., ODEN, D., \& WHITE, C. T. $V$ isually evoked cortical potentials and reaction time in relation to site of retinal stimulation. EEG Clinical Neurophysiology, 1967, 22, 313-324.

EASON, R. G., \& WHITE, C. T. Averaged occipital responses to stimulation of sites in the nasal and temporal halves of the retina. Psychonomic Science, 1967, 7, 309-310.

EASON, R. G., WHITE, C. T., \& ODEN, D. Averaged occipital responses to stimulation of sites in the upper and lower halves of the retina. Perception \& Psychophysics, 1967, 2, 423-425.

HARTER, M. R. Evoked cortical responses to checkerboard patterns: Effect of check-size as a function of retinal eccentricity. Vision Research, in press.

HARTER, M. R., \& SUITT, C. D. Visually-evoked cortical responses and pattern vision in the infant: A longitudinal study. Psychonomic Science, 1970, 18, 235-237.

HARTER, M. R., \& WHITE, C. T. Effects of contour sharpness and check-size on visually evoked cortical potentials. Vision Research, $1968,8,701-711$

HARTER, M. R., \& WHITE, C. T. Evoked cortical responses to checkerboard patterns: Effect of check-size as a function of visual acuity. EEG Clinical Neurophysiology, 1970, $28,48-54$

JEFFREYS, D. A. Separable components of human evoked responses to spatially patterned visual fields. EEG Clinical Neurophysiology, 1968, 24, 596. (Abstract)

JOHN, E. R., HERRINGTON, R. N., \& SUTTON, S. Effects of visual form on the evoked response. Science, 1967, 155, 1436-1442.

MackAY, D. M. Evoked brain potentials as indicators of sensory information processing. Neurosciences Research Program Bulletin, $1969,7,211-216$.

RIETVELD, W. J., TORDOIR, W. E., HAGENOUW, J. R., LUBBERS, J. A., \& SPOOR, Th. A. C. Visual evoked responses to blank and to checkerboard patterned flashes. Acta Physiol. Pharmacol. Neerl., 1967, 14, 259-285.

SPEHLMANN, R. The averaged electrical responses to diffuse and to patterned light in the human. EEG Clinical Neurophysiology, $1965,19,560-569$.

\section{The orienting reflex to changes in a conceptual stimulus dimension*}

\section{R. M. YAREMKO, MARJORIE W. BLAIR, and BRUCE T. LECKART San Diego State College, San Diego, Calif. 92115}

The galvanic skin response (GSR) component of the orienting reflex was recorded for four groups of $12 \mathrm{Ss}$ each. They were given 10 habituation trials to black on white numeric stimuli presented in seriatum. On Trial 11 an out-of-sequence test stimulus (TS) was delivered and was followed by one additional in-sequence number. The TS was either \pm 1 or \pm 11 places out of sequence. It was found that GSR magnitude to the TS was primarily a function of the amount of disparity provided by the TS, irrespective of the direction of that disparity. Dishabituation to the subsequent in-sequence stimulus was not, however, clearly present. The results were related to Sokolov's neuronal model of habituation and to previous research.

According to Sokolov (1963), magnitude of the orienting reflex (OR) is primarily a function of the amount of

* Supported in part by a grant to the first author from the San Diego State College Foundation. Requests for reprints should be addressed to R. M. Yaremko, Department of Psychology, San Diego State College, San Diego, California 92115 . change in the parameters of ambient stimulation when directional dynamogenic effects are not operative. Much support for this contention has been reported (e.g., Kimmel, 1960; Zimny \& Schwabe, 1965; Yaremko, 1969).

Other recent investigations (Unger, 1964: Zimny, Pawlick, \& Saur, 1969) indicate that the stimulus parameters of 